\title{
Descripción de la técnica de Rhoton modificada para la preparación de encéfalos en cadáveres y su práctica en el adiestramiento neuroquirúrgico en Chile
}

\section{"Rhoton's technique description for cadaveric preparations, modified to Chile's reality and applications in neurosurgical training"}

\author{
Tomás Poblete Poulsen ${ }^{1,2,3,4}$, Miguel Soto Vidal', Daniel Casanova Martinez ${ }^{1,5}$, Ximena Rojas Segura' \\ ${ }^{1}$ Laboratorio de Anatomía. Departamento de Anatomía y Medicina Legal, Facultad de Medicina, Universidad de Chile. \\ ${ }^{2}$ Servicio de neurocirugía. Hospital de Urgencia Asistencia Pública. \\ ${ }^{3}$ Servicio de neurocirugía. Hospital Clínico San Borja Arriarán. \\ ${ }^{4}$ Servicio de neurocirugía. Clínica Santa María. \\ ${ }^{5}$ Escuela de medicina, Universidad de Valparaíso, Campus San Felipe.
}

\section{Resumen}

Introducción: Los avances de la microcirugía y la implementación de nuevas técnicas neuroquirúrgicas hacen necesario que los neurocirujanos adquieran un mayor conocimiento de la neuromicroanatomía. Esto puede lograrse de mejor forma con el trabajo en especímenes cadavéricos en el laboratorio. Su estudio se hace más comprensible con una buena técnica de preservación cadavérica que permita mantener los tejidos biológicos por más tiempo, con una buena consistencia y con la inyección de silicona de color que permita distinguir claramente las estructuras venosas de las arteriales simulando mejor las condiciones de la realidad. Materiales y Métodos: Se utilizó una mezcla de solución conservadora compuesta por fenol, alcohol etílico y formalina para preservar las cabezas cadavéricas. Después de un período de tres semanas se realizó la inyección del material donde se administró una mezcla de silicona de color azul al sistema venoso y rojo al arterial. Las etapas de este proceso se describen detalladamente. Resultados: Desde el año 2014, un total de tres cabezas y un miembro superior se han preparado con la técnica descrita con resultados satisfactorios. Con el uso de material fotográfico se ilustran sus resultados. Se mencionan además las distintas variables involucradas en la obtención de una buena inyección. Conclusión: La preparación cadavérica como la antes descrita es un trabajo laborioso, pero que permite la obtención de preparados anatómicos de buena calidad que posibilitan su uso por mucho tiempo y permite distinguir claramente el sistema arterial del venoso.

Palabras clave: Laboratorio de neurocirugía, cabeza cadavérica, microcirugía, inyección silicona de color.

\section{Abstract}

Introduction: The advances of microsurgery and the introduction of new neurosurgical techniques make it necessary for neurosurgeons to acquire a greater knowledge of neuromicroanatomy. This can best be achieved by working on cadaveric specimens in the laboratory. Its study is made more meaningful with a good technique of cadaveric preservation that allows biological tissues to be maintained for a longer period of time, with a good tissue consistency and with the injection of a colored silicone mixture that allows to clearly distinguish between venous and arterial structures, simulating in a better way the condition of reality. Material and Method: A mixture of a preservative solution composed of phenol, ethyl

\section{Correspondencia a:}

Dr. Tomás Poblete Poulsen

Av. Santa María 0500, Providencia, Santiago, Chile.

tompoblete@gmail.com 
alcohol and formalin was used to preserve the cadaveric heads. After a period of three weeks, the venous system was injected with a blue silicone mixture and the arterial system with a red one. This process is described in a step-by-step format. Results: A total of three heads and an upper limb have been prepared since 2014 with the described technique with satisfactory results. Their results are shown with the use of photographic material. Different variables involved in obtaining a good injection process are also mentioned. Conclusions: The cadaveric preparation as the one described is a laborious work, but allows obtaining good quality anatomical preparations. The specimens can be used for a long period of time and it allows a clear distinction between venous and arterial structures.

Key words: Neurosurgery laboratory, cadaveric head, microsurgery, colored silicone injection.

\section{Introducción}

La implementación del microscopio quirúrgico en neurocirugía, el desarrollo de nuevos conceptos microquirúrgicos y el uso de técnicas de endoscopía, hacen fundamental que los residentes de cualquier programa de neurocirugía adquieran un mayor conocimiento de la neuromicroanatomía y puedan desarrollar nuevas habilidades técnicas durante su formación ${ }^{1}$. Si bien, los programas de formación de neurocirujanos en países desarrollados pueden contemplar el uso de microscopios 3D para la docencia, simuladores, resonancia nuclear magnética intra operatoria, neuro navegación y estereotaxia, por nombrar sólo algunos, su implementación es costosa y probablemente no sea la realidad actual de todos los centros formadores de neurocirujanos de nuestro país.

La facilidad en el acceso a la información que existe hoy con el uso de herramientas como internet, ha permitido un aumento del conocimiento que antes estaba restringido al pago de costosas suscripciones a revistas por lo general extranjeras. Por el contrario, las oportunidades de disección cadavéricas para el entrenamiento del neurocirujano siguen siendo limitadas en Chile y muchas veces asistir a cursos prácticos en el extranjero resulta costoso y son por períodos cortos de tiempo.

Si se considera que la mejor forma de aprendizaje de la neuromicroanatomía sigue siendo a partir del estudio de cadáveres humanos, la preparación de un espécimen bien conservado e inyectado es un paso fundamental para un estudio más comprensible y preciso de la neuroanatomía. Dada la normativa legal en muchos países y particularmente en el caso de Chile, probablemente la implementación de nuevas técnicas de preparaciones cadavéricas puedan desarrollarse en las facultades de medicina de nuestro país que tengan implementado un programa de donación de cuerpos con fines de investigación y académicos.

A continuación, describiremos nuestra técnica de preparación de cabezas cadavéricas que hemos implementado en el laboratorio de anatomía de la facultad de Medicina de la Universidad de Chile a contar del año 2014, que es el resultado de la implementación de la técnica aprendida por uno de los autores durante su entrenamiento en el laboratorio del Dr. Albert Rhoton Jr, en la Universidad de Florida, Gainesville. Se detallan además sus resultados y las distintas aplicaciones en la práctica actual.

\section{Materiales y Métodos}

\section{I) Procurar una cabeza cadavérica}

Dada la normativa legal actual en nuestro país que dificulta la obtención de piezas cadavéricas frescas de otros lugares, la existencia de un programa de donación de cuerpos asociado a una facultad de medicina es probablemente la forma que resulta más accesible para la obtención de piezas cadavéricas frescas.

El departamento de anatomía y medicina legal de la Facultad de Medicina de la Universidad de Chile, a través del proyecto desarrollado por Miguel Soto, tecnólogo médico y jefe del laboratorio, cuenta desde el año 2004 con un programa de donación de cuerpos para fines de investigación y académicos. Esta iniciativa cuenta actualmente con alrededor de 1.500 donantes en vida y un número de fallecimientos que fluctúa entre 26 y 28 personas al año?

Una vez que el donante fallece, su cuerpo es trasladado a las dependencias del departamento de anatomía de la Universidad de Chile. Si bien, el proceso de preservación en otros países se inicia con el embalsamamiento a través de la arteria femoral y el uso de una bomba de inyecciónaspiración que extrae la sangre e introduce una solución de embalsamamiento, nosotros en la Universidad de Chile al no disponer de estas bombas de inyección-aspiración nos cuestionamos la efectiva llegada del líquido de embalsamamiento a la cabeza cadavérica. Por esto es que apenas se dispone de un cuerpo se inicia la disección de ambas carótidas comunes a nivel cervical y ambas venas yugulares internas lo más cercano a la raíz del cuello posible. Luego de una arteriotomía y venotomía se introducen a través de los cuatro cabos craneales una sonda plástica lo más ancha posible, que se ajuste al lumen de la arteria y vena, y se fija con ligadura de seda 2.0. El cabo distal se liga con seda 2.0. A continuación y usando uno de los lados, se introduce con la ayuda de jeringas de $60 \mathrm{cc}$ la solución conservadora. Nuestra solución conservadora o de embalsamamiento está compuesta por fenol, alcohol etílico y formalina. Este proceso se realiza hasta que se logra extraer la mayor cantidad de sangre posible por los sistemas arterial y venoso. A continuación se ocluyen las cuatro sondas plásticas con distintas pinzas Kelly y se deja la cabeza y el cuerpo en una cámara fría por un período de alrededor de 2-3 semanas.

Transcurrido un período de 2-3 semanas, la cabeza es 
cortada con una sierra eléctrica lo más cercana a la raíz del cuello y es transferida al laboratorio de neuromicroanatomía.

\section{II) Inyección de silicona}

El proceso de inyección de la silicona de color es importante, porque permite distinguir fácilmente las estructuras venosas de las arteriales. Además, la repleción de las estructuras vasculares con silicona distiende sus paredes y las asemejan de mejor forma con las condiciones reales.

A continuación se detallan los elementos necesarios para la inyección con silicona de color:

1. Tijeras de macro y microcirugía.

2. Pinzas anatómicas y quirúrgicas.

3. Varias suturas de seda 2.0.

4. Varias pinzas Kelly.

5. Sondas plásticas de distintos lúmenes (Sondas de aspiración de distintos tamaños).

6. Puntas plásticas para micropipetas de colores azul y amarillo.

7. Motor eléctrico y fresas respectivas (en su lugar pueden usarse gubias).

8. 2 jeringas de $60 \mathrm{cc}$.

9. 2 vasos plásticos.

10. Silicona Dow Corning Xiameter RTV 3110.

11. Catalizador de silicona Dow Corning Xiameter RTV 3010 s y $f$ (slow y fast).

12. Adelgazador de silicona Dow Corning (200/50 fluid).

13. Pigmentos de color azul y rojo en polvo.

14. Pistola de calafatear.

Para la obtención de un resultado satisfactorio en cuanto a la inyección es importante seguir secuencialmente las siguientes etapas:

\section{1) Exposición de los grandes vasos}

Esta etapa se inicia exponiendo los 6 grandes vasos a nivel cervical: ambas carótidas comunes cervicales (recordar que la bifurcación carotidea ocurre a la altura de C4), arterias vertebrales y venas yugulares internas. En esta etapa y durante todo el proceso de disección, no es necesario disponer de un cabezal rígido tipo Mayfield, sino que con el uso de un picarón blando tipo herradura es suficiente para posicionar la cabeza de modo que el vértice quede apoyado sobre el soporte y sea la base del cuello la expuesta hacia el operador.

En este paso es conveniente retirar las sondas plásticas anteriormente colocadas y disecar detenidamente $2-3 \mathrm{~cm}$ de longitud de cada uno de los vasos. Mediante el uso de un motor eléctrico o gubias se amplían los forámenes transversos para exponer las arterias vertebrales (Figura 1).

\section{2) Intubación de los grandes vasos}

El objetivo de esta etapa es introducir una sonda plástica cuyo diámetro sea el mayor posible que se ajuste al lumen de cada uno de los vasos. Este hecho facilitará el proceso de irrigación y posterior inyección de la silicona. Los extremos de las sondas se fijan con ligaduras de seda 2.0 para evitar su desplazamiento en etapas posteriores (Figura 2).

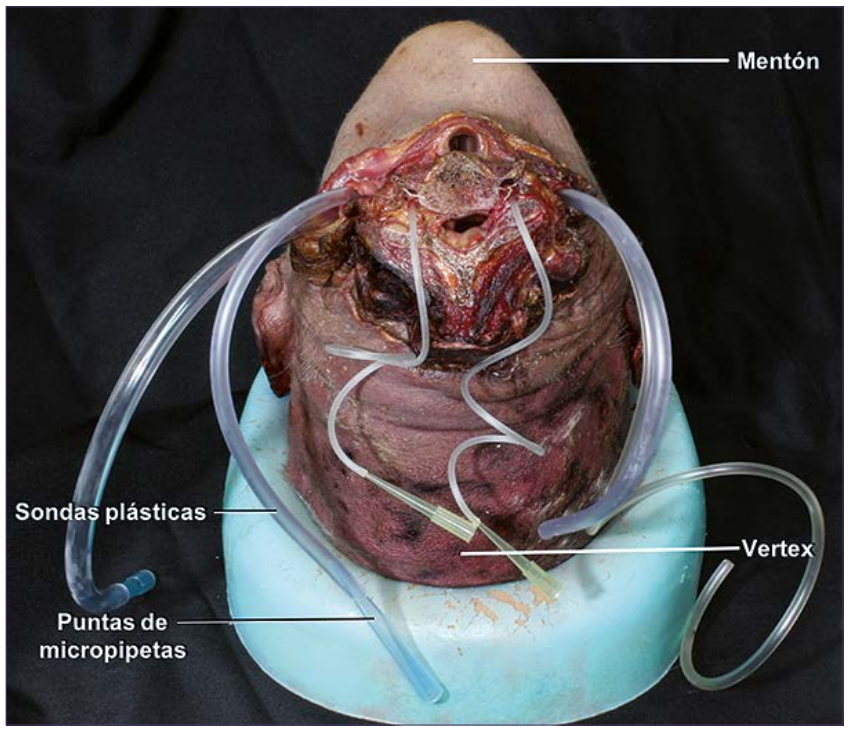

Figura 1. La cabeza cadavérica es apoyada sobre un picarón blando tipo herradura, se intubaron los 6 grandes vasos a nivel cervical y se han introducido sondas plásticas de distintos lúmenes a través de ellos ligándose sus extremos con sutura 2.0 para evitar su desplazamiento.

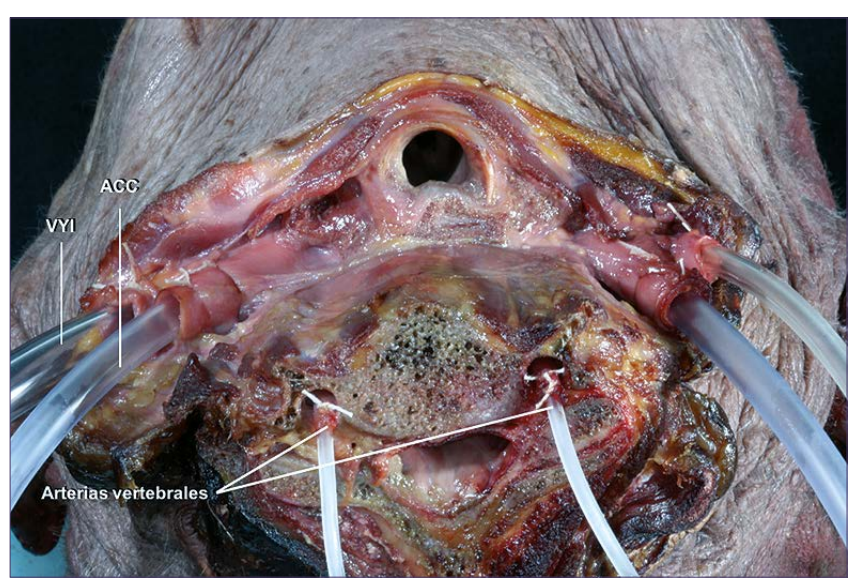

Figura 2. Con mayor magnificación se observan los 6 grandes vasos a nivel cervical con sus respectivas sondas plásticas. ACC: arteria carótida común, VYI: vena yugular interna, arterias vertebrales.

\section{3) Irrigación}

El objetivo de esta etapa es lavar profusamente cada uno de los 6 grandes vasos anteriormente identificados para remover la solución conservadora y todo tipo de coágulos presentes en el sistema vascular. Esta etapa es muy necesaria y es importante mencionar que una insuficiente irrigación permitirá que queden remanentes de coágulos en el interior de los vasos que resultará en una inyección de baja calidad.

Para este proceso se coloca la cabeza apoyada sobre el picarón en un fregadero y se llena un recipiente plástico con agua, idealmente tibia. Con el uso de puntas de micro pipetas de colores azul (para las sondas con mayor lumen) y amarillo (para las arterias vertebrales) utilizadas como conectores entre la sonda plástica y la jeringa de $60 \mathrm{cc}$, se lava por arrastre con agua hasta que el flujo de retorno por la sonda opuesta 
esté limpia de coágulos o detritos. Por lo general, cada ciclo de irrigación contempla lavar las arterias carótidas y venas yugulares con aproximadamente $1.000 \mathrm{cc}$ c/u y cada arteria vertebral con $500 \mathrm{cc}$ de agua tibia. Este proceso puede demorar aproximadamente 45-60 min. Se recomienda completar 2-3 ciclos al día y un total de 3 días seguidos de irrigación para asegurarse de que no queden restos de coágulos en el sistema vascular. Por lo general, el primer día resulta ser el más laborioso, porque el sistema vascular va disminuyendo su resistencia al paso del agua a medida que se limpia de coágulos en su interior. Esto mismo permite que la presión ejercida por el operador sea cada vez mayor y se logren liberar los coágulos más firmemente adheridos.

Durante el proceso de irrigación es importante detener cualquier fuga de líquido a nivel cervical que ocurra a través de vasos arteriales o venosos de mediano o gran calibre. Para esto pueden ligarse los vasos individualmente o con su musculatura para espinal con suturas de seda o bien, detener su salida con el uso de pinzas Kelly. La fuga de líquido a través de los plexos venosos de los forámenes transversos o del canal raquídeo es más difícil de controlar, pero puede detenerse también con el uso de oclusor con pinzas Kelly o bien con el uso de cera de hueso en algunos casos. La falla en controlar estas fugas de líquido a nivel cervical lleva a que posteriormente la silicona difunda por el espacio extra craneano en lugar del compartimento intracraneal. Si bien, es imposible controlar toda la salida de líquido a través de pequeños orificios, no debe existir salida activa y un chorro de agua para una buena inyección posterior de la silicona.

\section{4) Preparación de la mezcla de silicona de color}

La mezcla de silicona está compuesta por los siguientes productos:

1. Silicona Dow Corning Xiameter RTV 3110.

2. Catalizador de silicona Dow Corning Xiameter RTV 3010 s y $f$ (slow catalyst y fast).

3. Adelgazador de silicona Dow Corning (200/50 uid).

4. Pigmentos de color azul y rojo en polvo.

Las proporciones de silicona y del adelgazador de silicona son diferentes según se considere el sistema arterial o venoso. Como el sistema arterial ofrece mayor resistencia al paso de líquidos que el sistema venoso, la mezcla a preparar para el sistema arterial debe ser menos viscosa. Las proporciones de las mezclas de silicona a preparar según el sistema vascular son las siguientes:

- Para arterias = 2 adelgazador: 1 silicona

- Para venas = 1 adelgazador: 1 silicona

La cantidad de mezcla de silicona a preparar también es diferente. El sistema arterial requiere $60 \mathrm{cc}$-arterias carótidas $20 \mathrm{cc} \mathrm{c/u}$, arterias vertebrales $10 \mathrm{cc}$ c/u- y el venoso $120 \mathrm{cc}$ -venas yugulares $60 \mathrm{cc} c / \mathrm{u}$. Para asegurarse de que cada vaso sea inyectado con una cantidad suficiente, uno puede considerar elaborar 70-80 cc de mezcla para el sistema arterial y $125-150 \mathrm{cc}$ para el venoso.

A continuación, se prepara la mezcla de silicona y para ello se utilizan 2 vasos plásticos. En uno de ellos se vierte primero la silicona con el adelgazador de silicona siguiendo las proporciones antes indicadas según el sistema vascular a inyectar y se revuelve con un palo de madera. Posteriormente, se agrega el pigmento de color y se revuelve hasta obtener una mezcla homogénea. La cantidad de color es variable y según sea el gusto del operador, pero en general uno vierte 4-5 cucharadas de té llenas del pigmento. La mezcla se deja reposar un par de minutos para que pierda sus grumos de aire. Inmediatamente antes de la inyección de cada sistema vascular debe agregarse el catalizador de silicona-s of, en una proporción de 1/3-1/2 del tubo por cada 100-150 cc de mezcla de silicona.

\section{5) Inyección de la mezcla de silicona de color}

El objetivo de este paso es inyectar al sistema arterial y venoso con una cantidad adecuada de mezcla de silicona de color.

Si bien esta etapa puede realizarse con un solo operador, nosotros recomendamos que sea hecho por dos y por lo general se realiza al día siguiente o subsiguiente de haber finalizado el proceso de irrigación. La cabeza es puesta en el fregadero al interior de dos bolsas de basura, ya que este paso por más cuidado que uno tenga siempre deja algunos lugares manchados con silicona. Se requiere una pistola para calafatear y varias pinzas Kelly para ocluir cualquier fuga de la mezcla de silicona a través del cuello.

Por lo general, nosotros comenzamos inyectando el sistema arterial. Se ocluyen con las pinzas Kelly todas las sondas, excepto aquella que se usará para comenzar la inyección. Uno de los operadores coloca la jeringa con la mezcla de silicona en la pistola para calafatear y va apretando del gatillo lenta, pero continuamente. El asistente es el encargado de ocluir las fugas de silicona y de mantener la conexión entre las puntas de micro pipetas y las sondas plásticas, pues a medida que avanza el procedimiento la presión ejercida en el sistema es cada vez mayor y la conexión puede desplazarse. En algún punto durante la inyección, la presión ejercida es muy alta como para seguir con el proceso, por lo que se libera la oclusión de la sonda opuesta. Por ejemplo, si la inyección se inicia a través de la arteria carótida común derecha y se alcanza mucha resistencia en el sistema, se libera la oclusión colocado en la carótida común izquierda y se continúa ejerciendo presión con la pistola de calafatear hasta observar la emergencia de mezcla de silicona roja por la carótida común izquierda. Luego se ocluyen ambas sondas plásticas y se procede a inyectar el siguiente vaso. No hay que desanimarse si durante la inyección de un vaso uno no logra ver la emergencia de silicona por el vaso opuesto, pues aun así puede lograrse una buena inyección. Este proceso se repite para los 6 grandes vasos.

El procedimiento para inyectar el sistema venoso es similar. Nosotros tomamos la precaución de preparar la mezcla de silicona azul una vez inyectado el sistema arterial y por lo general preparamos la mezcla de un solo lado primero (aproximadamente $75 \mathrm{cc}$ para asegurarse), pues la viscosidad de esta mezcla es mayor y luego de agregado el catalizador se deben optimizar los tiempos. Sin embargo, esto último es una modificación de nuestra institución, ya que según el fabricante el catalizador requiere de 5-12 horas de acción para secar la silicona ${ }^{3}$.

\section{6) Guardado del espécimen cadavérico}

Luego de finalizado el proceso de inyección, la cabeza es guardada junto con sus sondas plásticas y sus respectivos 


\section{Artículo Original}

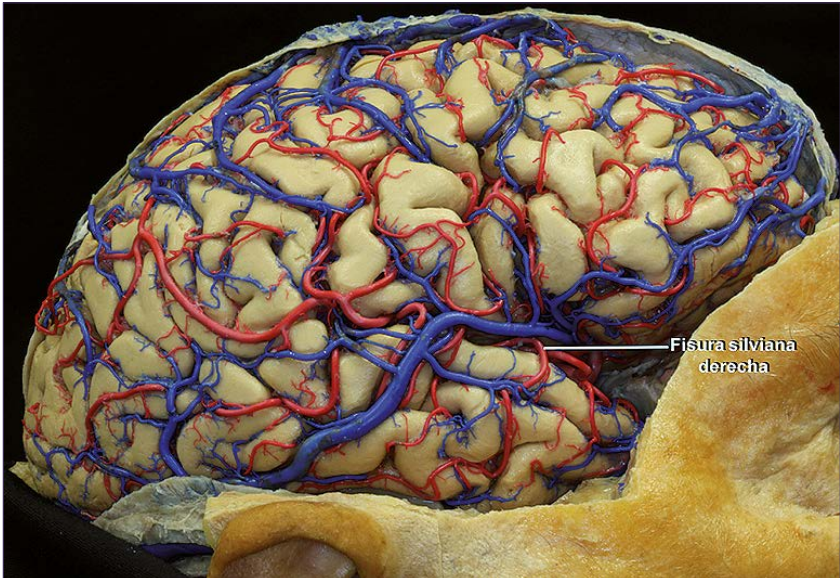

Figura 3. Imagen sagital del hemisferio cerebral derecho que demuestra una adecuada inyección de silicona de los sistemas arterial y venoso.

oclusores dentro de las bolsas de basura. Se espera un período de 24-48 horas antes de sacarlas de allí.

\section{7) Mantenimiento en el laboratorio}

Las cabezas son retiradas de las bolsas de basura donde se guardaron, se retiran las pinzas Kelly y se cortan las sondas plásticas.

Existe controversia en relación a la mejor forma de almacenar las cabezas para una mayor duración. El formaldehido se considerada la solución conservadora de elección, ya que destruye organismos vivos (incluso el $\mathrm{VIH}$ ) y previene el crecimiento bacteriano. Sin embargo, una de las mayores desventajas además de su penetrante olor y toxicidad es la rigidez que adquieren los tejidos. La retracción del parénquima cerebral se hace muy dificultosa, por lo que uno termina dañando las estructuras para demostrar una determinada estructura anatómica. Por todas estas razones es que nosotros almacenamos las cabezas en baldes plásticos sumergidas en una mezcla de etanol al $66 \%$. Esta solución de etanol al $66 \%$ permite mantener una buena consistencia del parénquima cerebral, evita el crecimiento microbiano y no tiene la toxicidad del formaldehido. Desde la primera inyección en el año 2014 hasta la actualidad, los especímenes se preservan en buenas condiciones sumergidos en esta solución.

\section{Resultados}

Desde el año 2014 cuando se inició la implementación de esta técnica de preparación cadavérica, se han inyectado 3 cabezas y hemos modificado la técnica para preparar también 1 miembro superior. Evidentemente, no todos los donantes en vida del programa de donación de cuerpos de la Universidad de Chile y que fallecen anualmente, logran ser preparados con esta técnica, pues una proporción de ellos son excluidos por presentar antecedentes de enfermedades cerebrovasculares y especialmente porque el recurso humano y materiales necesarios para esta preparación son escasos y han tenido que ser autofinanciados.

Sin embargo, los resultados de las inyecciones han

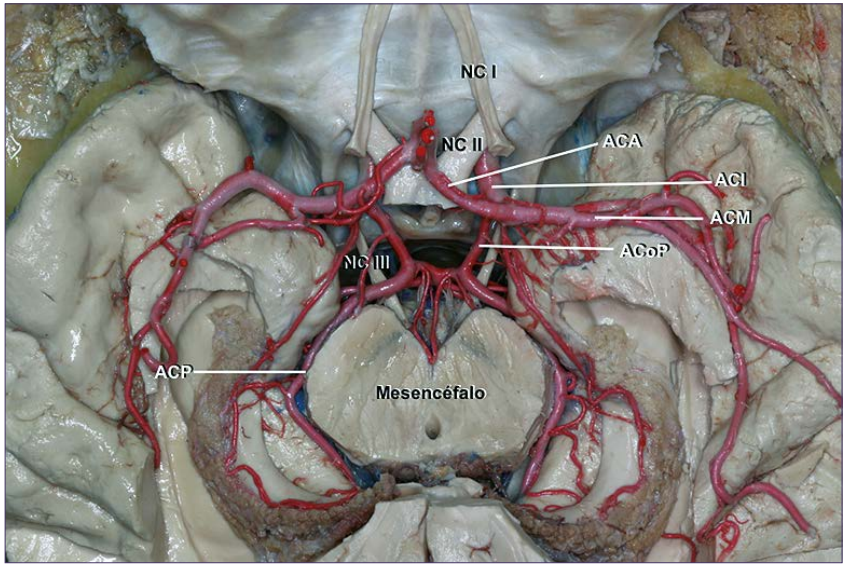

Figura 4. Imagen axial, proyección superior de la irrigación arterial de la base de cráneo. Se observa la composición de la circulación anterior y posterior.

sido auspiciosos. Es importante mencionar que en uno de los especímenes la mezcla de silicona azul no se secó por completo, sino que se mantuvo líquida. Probablemente esto se debió a haberle agregado poco catalizador a la mezcla de silicona azul. Por esta razón nosotros preferimos agregar $1 / 2$ tubo de catalizador-slow por cada 100-150 cc de mezcla de

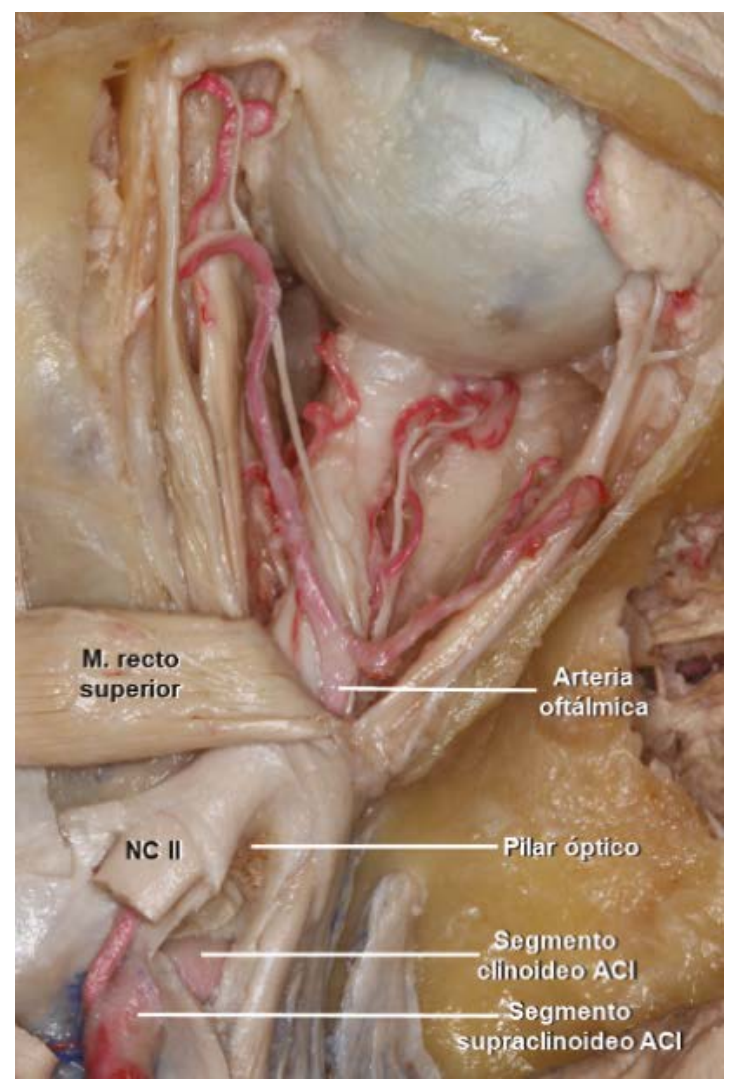

Figura 5. Proyección superior de la órbita derecha. Se ha eliminado la grasa orbitaria para demostrar los principales estructuras arteriales y neurales que la componen, así como también aquellas que atraviesan el canal óptico y fisura orbitaria superior. 


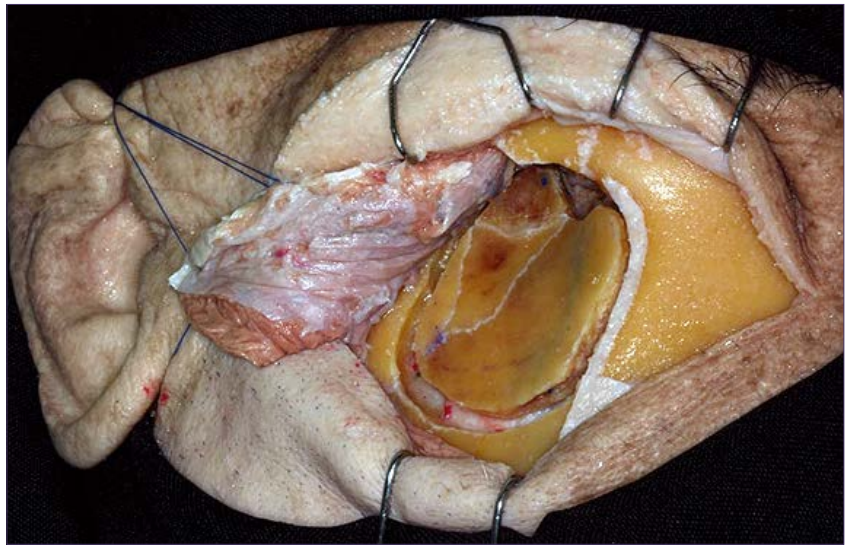

Figura 6. Acceso mini pterional izquierdo. Se aprecia una buena calidad de la piel y otras estructuras.

silicona y en algunas oportunidades hemos agregado también una pequeña cantidad de catalizador-fast (el productor vende también un catalizador-fast). Además, preparamos la mezcla de silicona para el sistema venoso en 2 etapas: primero hacemos $75 \mathrm{cc}$ de mezcla de silicona azul con su catalizador para inyectar un lado y luego los restantes $75 \mathrm{cc}$ de mezcla con su catalizador para inyectar el lado opuesto. Esta modificación de la técnica debe ser evaluada cuando tengamos un mayor número de inyecciones cadavéricas realizadas.

Los resultados de las preparaciones cadavéricas y los registros fotográficos de algunas de las disecciones realizadas se demuestran a continuación. En la Figura 3 se observa una adecuada inyección del sistema vascular arterial y venoso con buena solidificación de la silicona. La Figura 4 corresponde a una disección del sistema vascular arterial que ha sido regularmente usada para docencia a nivel de pre y postgrado. La Figura 5 corresponde a un proyecto elaborado para demostrar la micro anatomía de la órbita y que forma parte de un capítulo de un libro que está próximo a ser publicado por Ediciones "Journal". Por último, y en conjunto con el equipo de neurocirugía vascular y base del cráneo del Instituto de Neurocirugía Asenjo, uno de los últimos especímenes fue utilizado para demostrar secuencialmente la técnica del acceso "mini pterional" como modificación del acceso a través del "pterion" tradicional (Figura 6), lo que demuestra la importancia de trabajar en equipo y correlacionar la innovación en lo clínico con la práctica en el laboratorio.

\section{Discusión}

El cuerpo humano ha sido por siglos motivo de interés para la humanidad y probablemente la forma más sencilla de estudiarlo sea examinando el cuerpo de personas recién fallecidas. Sin embargo, el uso de cuerpos no preservados expone al operador a tejidos que con el paso del tiempo se descomponen y putrefaccionan ${ }^{4}$. Para lograr preservar los tejidos por más tiempo y desarrollado en buena parte por creencias religiosas, distintas civilizaciones de la antigüedad desarrollaron diferentes métodos de momificación. Los egip- cios utilizaron resinas de pino con las que impregnaron los vendajes con los que envolvían a sus cadáveres, pues postulaban que la vida eterna se asociaba con la preservación del cuerpo humano ${ }^{4,5}$. Otra de las culturas más antiguas en desarrollar la técnica de momificación fue la Chinchorro en la costa norte de nuestro país y sur del Perú. Estos últimos como parte de una manifestación compleja de un culto a la muerte y a los antepasados.

Con el tiempo distintos componentes han sido utilizados para preservar mejor los tejidos, pero fue el desarrollo del formaldehido hacia el siglo XIX el que es considerado en la actualidad el químico preservante de elección. Su disolución acuosa al $40 \%$ es conocida como formol o formalina y posee excelentes propiedades desinfectantes y de conservación de muestras biológicas. Sin embargo, tiene desventajas significativas como su penetrante olor y toxicidad si el operador se expone por períodos prolongados de tiempo. Provoca además una rigidez indeseada en la consistencia de los tejidos que limita el valor educacional de las preparaciones cadavéricas ${ }^{4}$. Por esta razón, nosotros en el laboratorio de anatomía de la Universidad de Chile utilizamos una solución conservadora compuesta por fenol, alcohol etílico y formalina en menores proporciones que ha demostrado tener mejor perfil de bioseguridad, mantiene sus propiedades bactericidas y la consistencia blanda de los tejidos.

Además de la preservación del material cadavérico, creemos que la técnica de inyección de los sistemas vasculares arterial y venoso con una mezcla de silicona de colores rojo y azul posee un enorme potencial educativo ${ }^{4,6}$. Con su aplicación es posible distinguir fácilmente las estructuras venosas, arteriales y neurales, hecho que en la práctica clínica es imprescindible para realizar una cirugía segura en nuestros pacientes.

El proceso de preparación cadavérica es sin duda un proceso laborioso que conlleva ensayo y error. Los protocolos de preparación cadavérica como el antes descrito, permiten establecer una forma de trabajo común, pero los resultados obtenidos son a veces desalentadores. Distintas variables están involucradas en el resultado final del proceso. Desde la calidad del espécimen (joven con una supuesta vascularización cerebral en buenas condiciones o anciano y un sistema vascular con mayor resistencia o estenosis), el tamaño del espécimen que implica ajustar la cantidad de la muestra a preparar o bien, la fuga de silicona a través de pequeños vasos a través del cuello que no logró controlarse con oclusores durante el proceso de inyección y que hace que la silicona no llegue al compartimento intracraneal. Probablemente, uno de los factores más relevantes para la obtención de una buena inyección sea la remoción precoz de la sangre una vez ocurrido el fallecimiento. Por esta razón, trabajar en los laboratorios de anatomía de facultades de medicina que dispongan de un programa de donación de cuerpos permite acceder rápidamente al cuerpo y remover estos coágulos. La remoción de los coágulos es mucho más difícil una vez que éstos ya se solidificaron.

Es importante mencionar que los avances de la microcirugía moderna, las expectativas por parte de nuestros pacientes y de sus familias por la obtención de un buen resultado postquirúrgico y la cada vez mayor exposición del médico a potenciales conflictos médico-legales, hacen imprescindible 
que el neurocirujano en formación o aquel que quiera continuar perfeccionándose disponga de un tiempo reservado de trabajo en un laboratorio de neuro micro anatomía. Creemos que sólo de esta forma se logra adquirir la tridimensionalidad de las estructuras anatómicas necesarias y acelerar la curva de aprendizaje de técnicas quirúrgicas más complejas para su posterior aplicación en la práctica clínica habitual.

Si bien, el desarrollo e implementación de un laboratorio de neuromicroanatomia toma tiempo y algunos elementos pueden ser costosos, creemos que este laboratorio dispone del elemento principal para trabajar que es la disponibilidad de material cadavérico fresco y en buenas condiciones. De esta forma, nuestro trabajo y esfuerzo, a pesar de todas las dificultades, ha tenido resultados satisfactorios y prometedores.

\section{Conclusiones}

En la actualidad, el fácil acceso a la información científica existente contrasta con las limitadas oportunidades de trabajo con especímenes cadavéricos en el laboratorio. A pesar de distintas herramientas como simuladores que buscan complementar la formación del neurocirujano, nada ha logrado reemplazar el estudio cadavérico en laboratorio.

Nuestro aporte es que la técnica de preparación cadavérica antes descrita puede ser una ayuda para el establecimiento de nuevos laboratorios a lo largo del país y mejorar la calidad de los preparados anatómicos y con esto contribuir con el desarrollo docente de las futuras generaciones de neurocirugía.

\section{Agradecimientos}

- Dr. Albert Rhoton $\mathrm{JR}^{\dagger}$, por sus enseñanzas y eterna generosidad.

- Marcelo Fuentes, tecnólogo de laboratorio, por la ayuda en la preparación y conservación del material cadavérico utilizado en este trabajo.

- Por sobre todo, a todas aquellas personas que en vida decidieron donar su cuerpo de forma generosa y desinteresada a la Universidad de Chile, con fines académicos y que hacen todo esto posible.

\section{Referencias}

1. Smith A, Gagliardi F, Pelzer NR, et al. Rural neurosurgical and spinal laboratory setup. J Spine Surg. 2015 Dec;1(1):57-64.

2. Mujica F. ¿Ser útiles después de morir? La decisión de donar el cuerpo para aportar a la formación de profesionales de la ciencia. 13 de enero 2019. Emol. Recuperado de http://www.emol. com/

3. Silastic RTV-3110 Mould-Making Base, Technical Data Sheet, Trademark of the Dow Chemical Company.

4. Sanan A, Abdel Aziz KM, Janjua RM, van Loveren HR, Keller JT. Colored silicone injection for use in neurosurgical dissections: anatomic technical note. Neurosurgery. 1999 Nov;45(5):1267-71; discussion 1271-4.

5. Brenner E. Human body preservation - old and new techniques. J Anat. 2014 Mar;224(3):316.44.

6. Shimizu S, Tanaka R, Rhoton AL Jr, et al. Anatomic dissection and classic three-dimensional documentation: a unit of education for neurosurgical anatomy revisited. Neurosurgery. 2006 May;58(5):E1000; discussion E1000. 